\title{
Fabrication of Comb-type self-oscillating gels by atom transfer radical polymerization for controlling autonomous swelling/deswelling behavior
}

\author{
Won Seok Lee', Takafumi Enomoto', Aya Mizutani Akimoto ${ }^{1}$ and Ryo Yoshida (i)
}

\begin{abstract}
In this study, we show the controlled self-oscillation behavior (i.e., the normalized amplitude, swelling rate, and deswelling rate) of self-oscillating gels by introducing grafted chains. The grafted chain was introduced to the base gel network by the "grafting from" method and atom-transfer radical polymerization (ATRP). We demonstrated that the properties of the grafted chain, including the monomer composition and length of the chain, can lead to notable selfoscillation behavior. The strategy in this study can pave the way for realizing the desirable operational functions of biomimetic actuators.
\end{abstract}

\section{Introduction}

Stimuli-responsive polymer gels mechanically respond to external signals, including temperature, $\mathrm{pH}$, light, electricity, and molecules. Therefore, the on-off switching of external signals allows stimuli-responsive polymer gels to be utilized as biomimetic actuators in smart materials ${ }^{1,2}$. In contrast, self-oscillating gels, developed by our group, have shown high potential for application as novel and smart soft materials. These self-oscillating gels display autonomous swelling-deswelling oscillation behavior without any external stimuli. The fundamental component of the self-oscillating gel is poly $(N$-isopropylacrylamide) (pNIPAAm) and the tris $\left(2,2^{\prime}\right.$-bipyridine $)$ ruthenium complex $\left(\mathrm{Ru}(\mathrm{bpy})_{3}{ }^{2+}\right)$. The former gives the whole gel thermosensitivity because it has a lower critical solution temperature. The latter is introduced into the gel network and acts as a catalyst for the Belousov-Zhabotinsky (BZ) reaction, a well-known oscillatory chemical reaction. During the oxidation of an organic substrate (e.g., malonic acid (MA)) by an oxidizing agent (e.g., sodium bromate) in the presence of the $\mathrm{Ru}(\mathrm{bpy})_{3}$ catalyst under acidic conditions, the $\mathrm{Ru}(\mathrm{bpy})_{3}$ in the selfoscillating gel undergoes periodic redox changes between its

\footnotetext{
Correspondence: Ryo Yoshida (ryo@cross.t.u-tokyo.ac.jp)

${ }^{1}$ Department of Materials Engineering, School of Engineering, The University of Tokyo, 7-3-1 Hongo, Bunkyo-Ku, Tokyo 113-8656, Japan
}

reduced $\left(\mathrm{Ru}(\mathrm{bpy})_{3}{ }^{2+}\right)$ and oxidized $\left(\mathrm{Ru}(\mathrm{bpy})_{3}{ }^{3+}\right)$ states. When $\mathrm{Ru}(\mathrm{bpy})_{3}{ }^{2+}$ is oxidized, the hydrophilicity and volume phase transition temperature (VPTT) of the gel network increase, resulting in a higher swelling ratio than in the reduced state. As a result, the self-oscillating gel exhibits autonomous and cyclic volumetric oscillation behavior during the $\mathrm{BZ}$ reaction ${ }^{3,4}$. We have recently broadened the applicability of these self-oscillating gels, which exhibit living organism-like autonomous movements, by fabricating various designs of self-oscillating gel actuators, including a selfwalking gel $^{5}$; a self-regulated conveyor ${ }^{6}$; ciliary motion actuators, such as villi ${ }^{7}$; and intestine-like tubular gels with peristaltic motion ${ }^{8}$, that exhibit different functions.

A significant challenge for the further application of selfoscillating gels is controlling the self-oscillating behavior, such as the period and volumetric amplitude. Changing the oscillation condition has been suggested to address this problem, including regulating the concentration of chemical substrates for the BZ reaction (e.g., sodium bromate or $\mathrm{MA})^{9}$ or the oscillation temperature ${ }^{10}$. Other alternatives include modulating the physical shape of the self-oscillating gel $^{5-9,11,12}$ and chemically modifying the gel network ${ }^{13}$. Moreover, we have reported comb-type self-oscillating gels by introducing grafted polymer chains to base gel networks ${ }^{14}$. Conventional comb-type self-oscillating gels were 
fabricated by the "grafting through" method using the free radical polymerization of poly(NIPAAm- $r$-Ru(bpy $)_{3}{ }^{2+}$ ) macromonomers. The comb-type self-oscillating gel consists of the main chain and grafted chain, both of which are poly(NIPAAm- $r-\mathrm{Ru}(\mathrm{bPy})_{3}{ }^{2+}$ ) with the same content of $\mathrm{Ru}$ (bpy) ${ }_{3}{ }^{2+}$. The freely mobile grafted polymer chains promote their quick responsiveness to the redox change of $\mathrm{Ru}$ (bpy) ${ }_{3}{ }^{2+}$, resulting in the rapid swelling-deswelling behavior of the whole gel network during self-oscillation. Therefore, the comb-type self-oscillating gel exhibits a larger amplitude than normal self-oscillating gels since the gel can sufficiently swell or deswell before the redox state changes. Nevertheless, the above approach does not offer controlled oscillation behavior because of the uncontrollability of the "grafting through" method. During polymerization, macromonomers can hinder another reactant (e.g., monomers and macromonomers) from getting close to the propagating oligomer chain, resulting in low reactivity due to steric hindrance ${ }^{15-18}$.

To overcome this problem, we designed comb-type selfoscillating gels fabricated by the "grafting from" method and atom transfer radical polymerization (ATRP). ATRP offers well-defined polymers with controlled molecular weights, molecular structures, and compositions by polymerizing various functional monomers ${ }^{17}$. In this study, we introduced graft chains into self-oscillating gels by ATRP from the initiator immobilized in the main chain of the base gel network. The comb-type gels were synthesized under various designed conditions (the composition of the graft chains and ATRP reaction time), and the swelling/deswelling kinetics and amplitude of the prepared comb-type self-oscillating gels were investigated.

\section{Materials and methods Materials}

$N$-Isopropylacrylamide (NIPAAm) was kindly provided by KJ Chemicals (Tokyo, Japan) and purified by recrystallization from toluene/hexane. $N$-(3-Aminopropyl)methacrylamide hydrochloride (NAPMAm) was purchased from Polyscience (Warrington, PA) and used as received. $N, N^{\prime}-$ Methylenebisacrylamide (MBAAm), ammonium persulfate (APS), $N, N, N^{\prime}, N^{\prime}$-tetramethylethylenediamine (TEMED), ethyl 2-bromoisobutyrate (EBiB), copper(I) bromide, dimethyl sulfoxide (DMSO) sodium bromate $\left(\mathrm{NaBrO}_{3}\right), 1 \mathrm{M}$ $\mathrm{HNO}_{3}$ aqueous solution and MA were purchased from Wako Pure Chemical Industries (Osaka, Japan). The water used in this study was purified by a water purifier (WA200, Yamato Scientific, Tokyo, Japan). 2-Bromoisobutanoic acid $\mathrm{N}$-hydroxysuccinimide ester (abbreviated as NHS-initiator) ${ }^{19}$ and rhodamine $\mathrm{B}$ hydroxyethyl methacrylate ester $(\mathrm{RhB})^{20}$ were synthesized according to previous works. Bis (2,2'-bipyridine) (1-(4'-methyl-2,2'-bipyridine-4-carbonyloxy)-2,5-pyrrolidinedione) ruthenium(II) ( $\left.\mathrm{Ru}(\mathrm{bpy})_{3}-\mathrm{NHS}\right)$ was purchased from Trylead Chemical (Hangzhou, China).
Glass capillaries (inner diameter $=850 \mu \mathrm{m}$ ) were purchased from Nichiden Rika Glass Co., Ltd. (Kobe, Japan).

\section{Preparation and characterization of the free polymers}

Poly(NIPAAm) and poly(NIPAAm- $r$-NAPMAm) were synthesized by ATRP. Briefly, a monomer solution $(45 \mathrm{~mL})$ containing NIPAAm and/or NIPAAm was degassed with Ar for $30 \mathrm{~min}$. The monomer solutions were prepared based on the recipes shown in Table S1. Then, $\mathrm{Me}_{6}$ TREN $(147 \mu \mathrm{L})$ and $\mathrm{CuBr}(71.7 \mathrm{mg})$ were added in order. An ATRP initiator solution composed of $\mathrm{EBiB}(17.1 \mathrm{mg})$, DMSO $(250 \mu \mathrm{l})$, and water $(5 \mathrm{ml})$ was also degassed by $\mathrm{Ar}$ bubbling for $30 \mathrm{~min}$. The ATRP initiator solution was added to the monomer solution in an Ar gas-filled plastic bag. The ATRP reaction times of each sample are also listed in Table S1. After the ATRP reaction, the reaction mixture was dialyzed against water for one week. The dialyzed polymer was obtained by freeze-drying. To conjugate $\mathrm{Ru}$ (bpy $)_{3}$ to the free polymer, the obtained free polymer was dissolved in DMSO solution containing $70 \mathrm{mM} \mathrm{Ru}(\mathrm{bpy})_{3}$ NHS and triethylamine, followed by stirring at $25^{\circ} \mathrm{C}$ for $24 \mathrm{~h}$. The polymer solution was dialyzed with deionized water and then recovered by freeze-drying. The monomer conversion, the number-average molecular weight, and NAPMAm composition in the polymers were evaluated by ${ }^{1} \mathrm{H}$ nuclear magnetic resonance (NMR) (JEOL, JLMLA400WB) using $\mathrm{D}_{2} \mathrm{O}$ as the solvent.

\section{Preparation of the poly(NIPAAm-r-NAPMAm) base gel}

The poly(NIPAAm- $r$-NAPMAm) base gel was synthesized by free-radical polymerization. Briefly, two solutions, a monomer solution composed of NIPAAm $(808 \mathrm{mg}$, $95 \mathrm{~mol} \%), \quad$ NAPMAm $\quad(26.8 \mathrm{mg}, 2 \mathrm{~mol} \%), \quad$ MBAAm (34.7 $\mathrm{mg}, 3 \mathrm{~mol} \%)$ and TEMED $(11.2 \mu \mathrm{L})$ in water $(4 \mathrm{ml})$ and an initiator solution of APS $(17.1 \mathrm{mg})$ in water $(1 \mathrm{ml})$, were prepared. Then, each solution was cooled to $0{ }^{\circ} \mathrm{C}$ in iced water, followed by an Ar bubbling process for degassing for $10 \mathrm{~min}$. After that, the initiator solution was poured into the monomer solution and immediately stirred by a vortex mixer. The pregel solution was placed into glass capillaries (inner diameter $=850 \mu \mathrm{m}$ ). Then, the loaded glass capillaries were maintained at $4{ }^{\circ} \mathrm{C}$ for $24 \mathrm{~h}$ of free radical copolymerization. After the gelation process, the loaded glass capillaries were immersed in distilled water at $50{ }^{\circ} \mathrm{C}$ to shrink the gels, allowing them to be removed from the glass capillaries by poking with a thin rod-type apparatus. The obtained gels were cut into $10 \mathrm{~mm}$ pieces and purified by dialysis against water for 1 week; the water was exchanged every day.

\section{Sample abbreviation}

The poly(NIPAAm- $r$-NAPMAm- $r$-NAPMAm $\left(\mathrm{Ru}(\mathrm{bpy})_{3}\right)$ ) gel is named the Base Gel. The comb-type self-oscillating gels are referred to as NINA_X_Y, where $\mathrm{X}$ indicates the 
molar composition of NAPMAm in the ATRP solution, and $\mathrm{Y}$ represents the ATRP reaction time. In addition, the samples with the same NAPMAm molar composition (X) and different ATRP reaction times $(\mathrm{Y})$ are comprehensively expressed as the NINA_X series.

\section{Immobilization of the ATRP initiator on the poly(NIPAAm- r-NAPMAm) base gel}

The fabricated poly(NIPAAm-r-NAPMAm) base gels were immersed in water $(7 \mathrm{ml})$. NHS-initiator $(17.1 \mathrm{mg})$ was dissolved in DMSO $(250 \mu \mathrm{l})$ to prepare an NHSinitiator solution. The NHS-initiator solution was poured into water containing the gels. The solution was stirred at $25^{\circ} \mathrm{C}$ for $24 \mathrm{~h}$. The initiator-immobilized gels were collected and purified by dialysis against water for one week; the water was exchanged every day.

\section{Fabrication of the comb-type gels}

The monomer solutions were prepared according to the recipe shown in Table S1. Each solution was degassed by Ar bubbling for $30 \mathrm{~min}$, followed by the sequential addition of $\mathrm{Me}_{6}$ TREN $(147 \mu \mathrm{L})$ and $\mathrm{CuBr}(71.7 \mathrm{mg})$. The initiator-immobilized poly(NIPAAm- $r$-NAPMAm) gels were placed in water, and the water was degassed by Ar bubbling for $30 \mathrm{~min}$. The gels were carefully moved to ATRP monomer solutions. It should be noted that the operation was conducted in a plastic bag filled with $\mathrm{Ar}$ gas. The ATRP reaction times of each sample are also listed in Table S1. After the ATRP reaction, the gels were dialyzed against water for 1 week; the water was exchanged every day.

\section{Fabrication of the self-oscillating comb-type gels}

The comb-type gels were immersed in superdehydrated DMSO $(1 \mathrm{ml})$ containing triethylamine $(13.9 \mu \mathrm{l})$ for one day. To conjugate $\mathrm{Ru}(\mathrm{bpy})_{3}$-NHS to the amino group of the gels, the gels were immersed in the superdehydrated DMSO $(0.5 \mathrm{ml})$ solution containing $\mathrm{Ru}(\mathrm{bpy})_{3}-\mathrm{NHS}$ $(70 \mathrm{mM})$ and triethylamine $(6.96 \mu \mathrm{l})$ at $45^{\circ} \mathrm{C}$ for $24 \mathrm{~h}$. Then, the $\mathrm{Ru}(\mathrm{bpy})_{3}$-conjugated gels were dialyzed against a water/DMSO mixture (1:1) for four days and water for the following 3 days.

\section{Measurement of the equilibrium swelling ratio}

The gel samples were equilibrated in water containing $894 \mathrm{mM} \mathrm{HNO}_{3}$ and $84 \mathrm{mM} \mathrm{NaBrO}_{3}$ for the oxidized state $\left(\mathrm{Ru}(\mathrm{bpy})_{3}{ }^{3+}\right)$ and $894 \mathrm{mM} \mathrm{HNO}_{3}, 64 \mathrm{mM} \mathrm{MA}$, and $84 \mathrm{mM} \mathrm{NaCl}$ for the reduced state $\left(\mathrm{Ru}(\mathrm{bpy})_{3}{ }^{2+}\right) . \mathrm{NaCl}$ was added to maintain the ionic strength. Photos of the gels in each redox state were recorded by an optical microscope (Keyence, VHX 900). The acquired images were analyzed with ImageJ software (NIH) to calculate the gel diameters at the various temperature points. The equilibrium swelling ratios were calculated by dividing the measured gel diameters $(d)$ by the inner diameter of the glass capillary $\left(d_{\mathrm{o}}, 850 \mu \mathrm{m}\right)$ utilized in fabricating the poly(NIPAAm- $r$ NAPMAm) base gel. The analyzed data were modeled by a Boltzmann sigmoidal equation (Eq. S1).

Measurement of the self-oscillation behavior and kinetics

The gels were immersed in a catalyst-free $\mathrm{BZ}$ solution containing $894 \mathrm{mM} \mathrm{HNO}_{3}, 84 \mathrm{mM} \mathrm{NaBrO}_{3}$, and $64 \mathrm{mM}$ malonic acid at $20^{\circ} \mathrm{C}$. The time profiles of the gel diameter during self-oscillation were observed from images recorded by an optical microscope (Keyence, VHX 900). Based on the gel diameter profiles (Fig. S1), the amplitude, swelling rates, and deswelling rates were calculated and plotted as a function of time. Note that the analysis methods for each component are provided in Fig. S2.

\section{Results}

Fabrication of the comb-type self-oscillation gels

Figure 1 schematically illustrates the fabrication process of the comb-type self-oscillating gels. First, the graft chains (poly(NIPAAm) or poly(NIPAAm-r-NAPMAm)) were introduced in the poly(NIPAAm- $r$-NAPMAm) base gel network. Then, $\mathrm{Ru}(\mathrm{bpy})_{3}$ was conjugated to the primary amines in NAPMAm. An example of the chemical structure of the comb-type self-oscillating gels is displayed in Fig. 2, in which poly(NIPAAm- $r$-NAPMAm) is grafted to the main gel network. The detailed fabrication process and chemical structure are displayed in Fig. S3.

\section{Controllability of ATRP for synthesizing poly(NIPAAm) and poly(NIPAAm-r-NAPMAm)}

In the beginning, we investigated the controllability of ATRP for synthesizing poly(NIPAAm) and poly (NIPAAm- $r$-NAPMAm). The ATRP reaction kinetics of the NINA series are shown in Fig. 3. The time course of monomer conversion (\%) and the number-average molecular weight $\left(M_{\mathrm{n}}\right)$ of the NINA series showed similar trends. In the NINA_0 series, the monomer conversion and $M_{\mathrm{n}}$ reached saturation in $1 \mathrm{~h}(97.7 \%$ conversion and $M_{\mathrm{n}}=11,052 \mathrm{kDa}$ ). In the case of the NINA_2 series, the time course of monomer conversion and $M_{\mathrm{n}}$ were slightly slower than that of the NINA_0 series and reached saturation in $5 \mathrm{~h}(92.5 \%$ monomer conversion and $\left.M_{\mathrm{n}}=10,585 \mathrm{kDa}\right)$. In the NINA_10 series, the monomer conversion and $M_{\mathrm{n}}$ grew much slower than those of the others. The monomer conversion and $M_{\mathrm{n}}$ of NINA_10_24 were $73.2 \%$ and $8772 \mathrm{kDa}$, respectively. We note that the monomer compositions under each reaction condition were consistent with the feeding ratio. All the calculated results are listed in Table S1. The successful conjugation of Ru (bpy) $)_{3}$ to the amine groups in NAPMAm was confirmed by NMR spectroscopy (Fig. S4). 


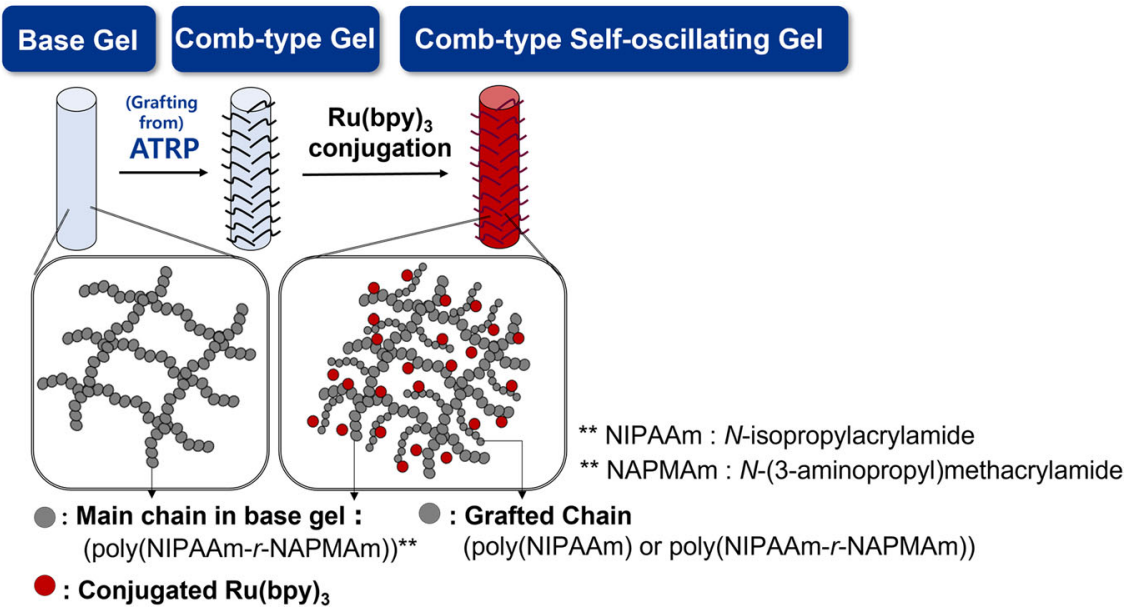

Fig. 1 The comb-type self-oscillating gels. Schematic illustration of the comb-type self-oscillating gels.

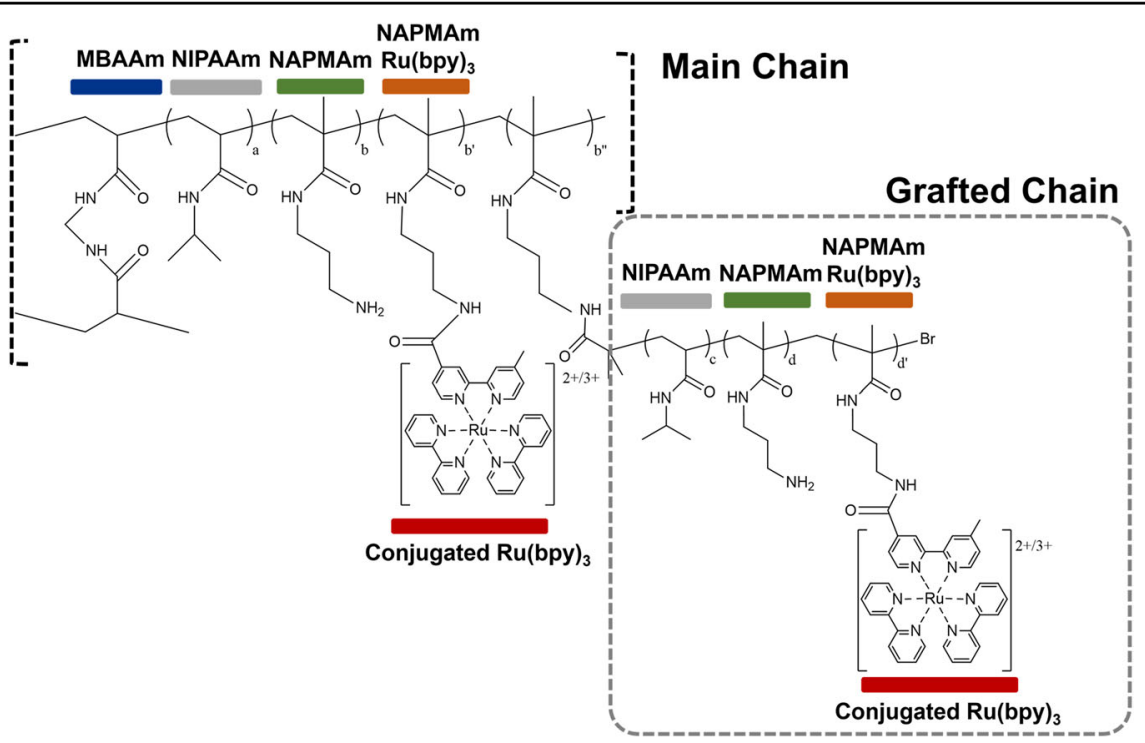

Fig. 2 The representative chemical structure of the comb-type self-oscillating gels. Chemical structure of the comb-type self-oscillating gels.

\section{Equilibrium swelling ratio}

Figure 4 displays representative data of the equilibrium swelling ratio of the Base Gel and NINA_10_24. The graphs of all samples are presented in Fig. S5. We fitted all the graphs using the Boltzmann sigmoidal equation (Eq. S1) to quantitatively calculate the difference in the equilibrium swelling ratio. Table $\mathrm{S} 2$ reveals the adjusted parameters of the fitted Boltzmann sigmoidal equations. Figure $4 \mathrm{a}, \mathrm{b}$ shows the measured and fitted plot of the equilibrium swelling ratio of the Base Gel and NINA_10_24 as a function of temperature. The gap of the equilibrium swelling ratio between the two redox states became larger in NINA_10_24 than in the Base Gel.
The differences in the equilibrium swelling ratio were maximized at approximately $20^{\circ} \mathrm{C}$ regardless of the grafted chain. Therefore, we decided on an observation temperature of $20^{\circ} \mathrm{C}$ for the self-oscillating experiments. Figure $4 \mathrm{c}$ indicates that the differences in the equilibrium swelling ratio between the two redox states at $20^{\circ} \mathrm{C}$ can be adjusted by varying the NAPMAm composition and ATRP reaction time. We note that the $y$-axis result where the $\mathrm{x}$-axis was zero represented the value of the Base Gel. The gap between the redox states became more significant in the comb-type self-oscillating gels, particularly as the NAPMAm composition increased and the ATRP reaction time became longer. 
(a)

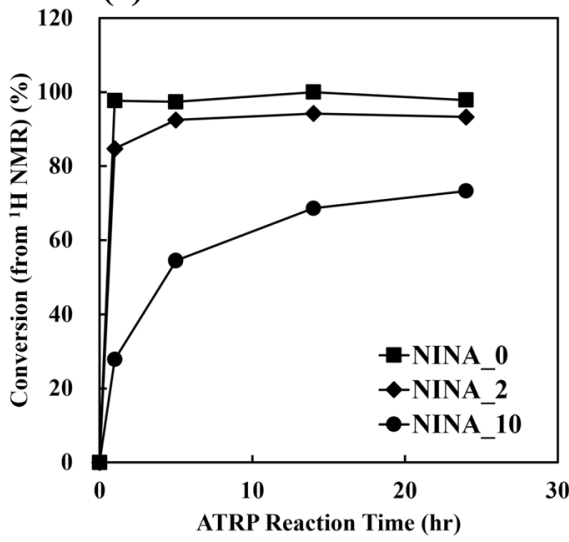

(b)

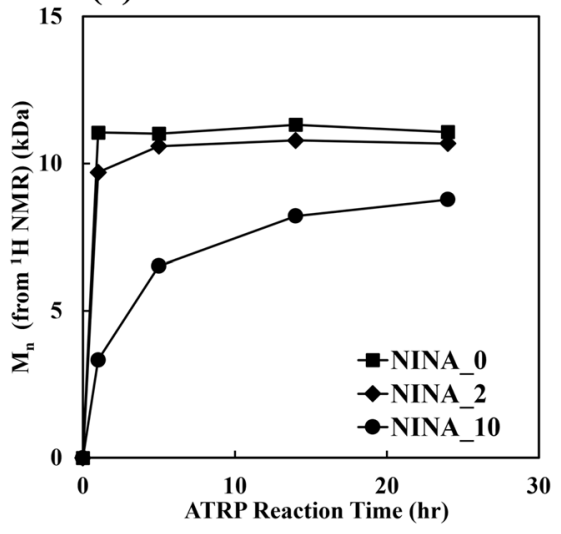

Fig. 3 Analysis of free-polymers synthesized using ATRP. Results of (a) monomer conversion (\%) and (b) $M_{n}$ of free-polymer synthesis with various NAPMAm compositions as a function of the ATRP reaction time. These values were observed from the ${ }^{1} \mathrm{H}$-NMR results.

(a)

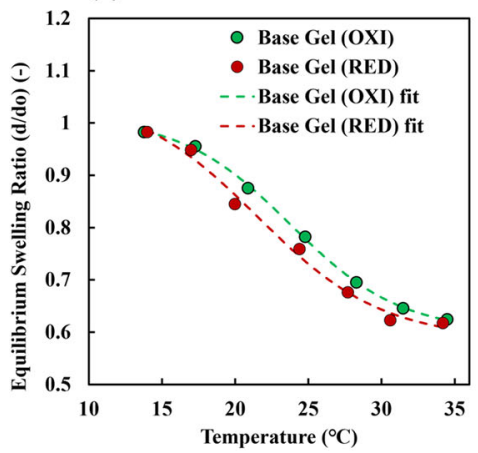

(b)

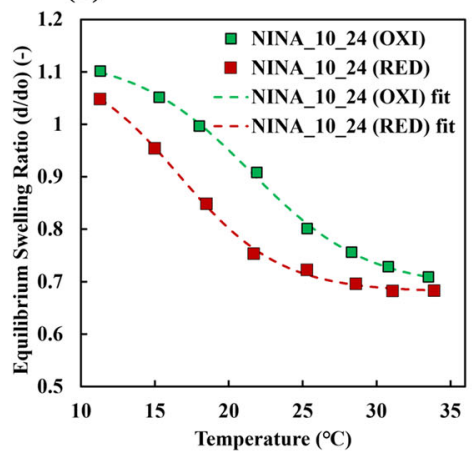

(c)

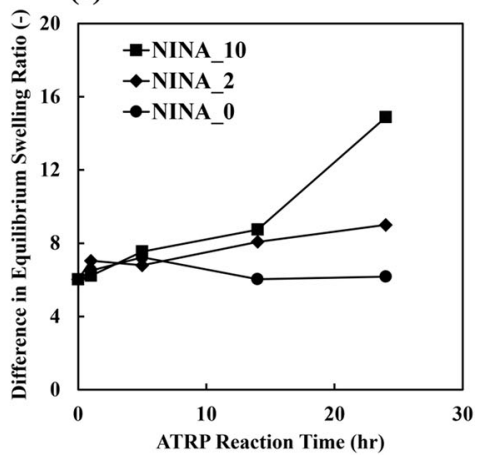

Fig. 4 Analysis of the equilibrium swelling ratio of the gels in each redox state. Equilibrium swelling ratio and fitting curve of the a Base Gel and b NINA_10_24. c Difference in the equilibrium swelling ratio between two redox states at $20^{\circ} \mathrm{C}$ observed from the fitted data (details are provided in the Supplementary Information). The oxidized state was conditioned with the substrate solution containing $\left[\mathrm{HNO}_{3}\right]=894 \mathrm{mM}$ and $\left[\mathrm{NaBrO}_{3}\right]=$ $84 \mathrm{mM}$. The reduced state was realized with the substrate solution containing $\left[\mathrm{HNO}_{3}\right]=894 \mathrm{mM},[\mathrm{NaCl}]=84 \mathrm{mM}$, and $[\mathrm{MA}]=64 \mathrm{mM}$.

\section{Self-oscillating behavior and kinetics}

Figure $5 \mathrm{a}, \mathrm{b}$ indicates the volumetric oscillation profile of the Base Gel and NINA_10_24 in the BZ substrate solution. (See Fig. S1 for the volumetric oscillation profiles of all samples). The normalized amplitude (Fig. 5c), swelling rate (Fig. 5d), and deswelling rate (Fig. 5e) of all samples during the $\mathrm{BZ}$ reaction were analyzed based on these gel diameter oscillation profiles. The $y$-axis value where the $x$-axis was zero corresponds to the result of the Base Gel. Figure $5 c$ shows the relationship between the ATRP reaction time and normalized amplitudes during the $\mathrm{BZ}$ reactions. The normalized amplitudes of the selfoscillation of the Base Gel were $1.83 \pm 0.11 \%$, and the NINA_0 series showed similar values compared to the Base Gel. The normalized amplitudes of the NINA_2 series were slightly larger than those of the Base Gel. Both the NINA_0 and NINA_2 series showed no dependencies between the normalized amplitudes and
ATRP reaction times. On the other hand, the normalized amplitudes of the self-oscillation of the NINA_10 series strongly depended on the ATRP reaction time. The normalized amplitude of NINA_10_24, which had the largest normalized amplitude, was 2.19 times larger than that of the Base Gel.

Figure $5 \mathrm{~d}$ shows the correlation between the swelling rate during self-oscillation and the ATRP reaction time. The swelling rate of the Base Gel was $3.50 \pm 1.20 \times 10^{-2}$ $\mu \mathrm{m} / \mathrm{s}$. The swelling rates of the NINA_0 series were slightly faster with the Base Gel, and the NINA_2 samples exhibited faster swelling rates. The swelling rates of the NINA_10 series increased with the ATRP reaction time, showing the fastest swelling rate in NINA_10_24, which was 3.14 times faster than that of the Base Gel.

Figure 5e shows the deswelling rate during selfoscillation as a function of the ATRP reaction time. The deswelling rate of the Base Gel was $2.60 \pm 0.10 \times 10^{-2} \mu \mathrm{m} / \mathrm{s}$. 
(a) Base Gel

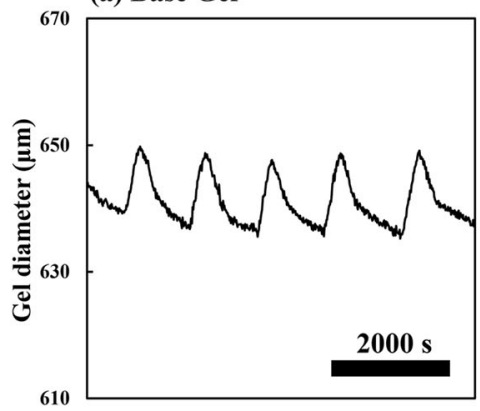

(c)

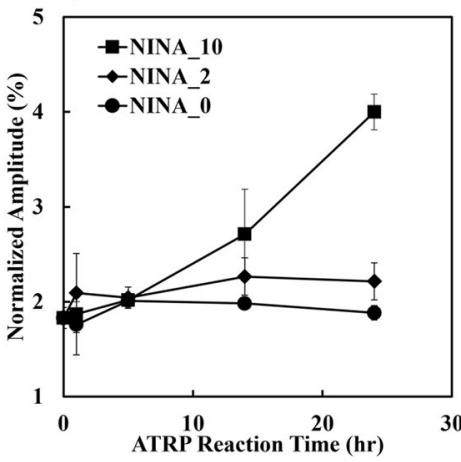

(b) NINA_10_24

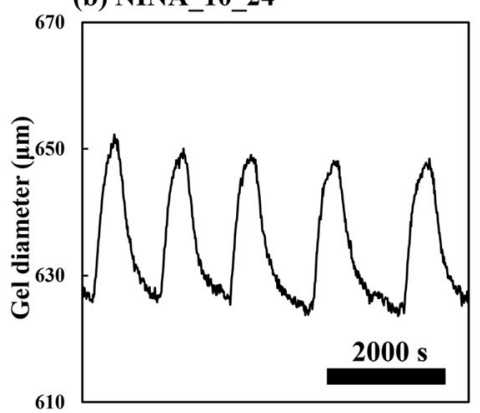

(d)

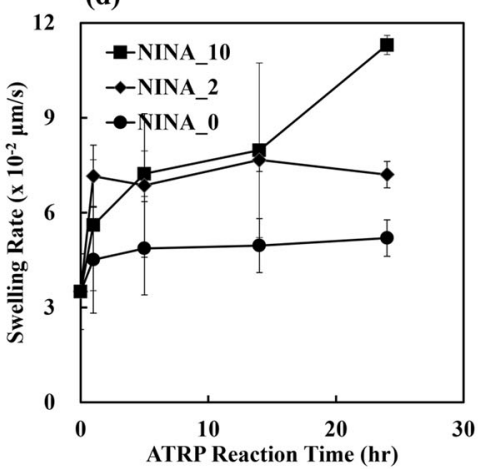

(e)

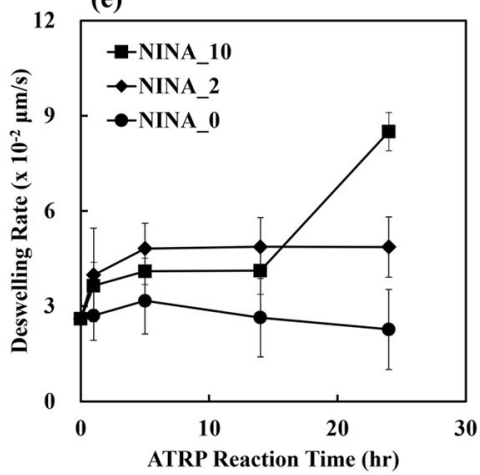

Fig. 5 Analysis of the self-oscillating behavior and kinetics during the BZ reaction. Time profiles of the gel diameter during self-oscillation of the $\mathbf{a}$ Base Gel and $\mathbf{b}$ NINA_10_24 at $20^{\circ} \mathrm{C}$. c Normalized amplitude, $\mathbf{d}$ swelling rate, and $\mathbf{e}$ deswelling rate of the various NAPMAm compositions calculated from the time profiles of the gel diameter plotted as a function of the ATRP reaction time. The substrate solution for self-oscillation contains $\left[\mathrm{HNO}_{3}\right]=894 \mathrm{mM},\left[\mathrm{NaBrO}_{3}\right]=84 \mathrm{mM}$, and $[\mathrm{MA}]=64 \mathrm{mM}$.

The deswelling rates of the NINA_0 series were almost identical to those of the Base Gel. The NINA_2 series displayed faster deswelling rates than the Base Gel and the NINA_0 series. The deswelling rates of the NINA_10 series exhibited a dependence on the ATRP reaction time. The fastest deswelling rate was shown in NINA_10_24, which was 3.14 times faster than that of the Base Gel.

\section{Discussion}

We assumed that one of the key factors to improve the autonomous oscillatory behavior of the self-oscillating gels during the $\mathrm{BZ}$ reaction was the swelling/deswelling kinetics. The cyclic redox changes of $\mathrm{Ru}(\mathrm{bpy})_{3}$ in the selfoscillating gels coupled with the BZ reaction drive their volumetric oscillations ${ }^{3,4}$. Thus, if the volumetric changes of the self-oscillating gels could not follow the cyclic redox changes induced by the BZ reaction due to the slow swelling/deswelling kinetics, the amplitude of the volumetric oscillations would be highly limited. From this point of view, we employed a strategy to improve the swelling/deswelling kinetics of self-oscillating gel by introducing grafted chains in the whole gel network. The grafted polymer chain moves faster than the fixed polymer chain in the leading gel network. A previous study reported that a grafted poly(NIPAAm) chain enhanced the shrinkage speed and amplitude of the main poly (NIPAAm) gel network induced by temperature changes $^{21,22}$. We also reported the self-oscillating behavior of comb-type self-oscillating gels, which were composed of poly(NIPAAm- $r$ - Ru(bpy $)_{3}$ ) main chains and poly (NIPAAm- $r$-Ru(bpy) $)_{3}$ ) graft chains ${ }^{14}$. The swelling/deswelling rate and amplitude were improved during the BZ reaction, resulting from the presence of the grafted chain. Although exploiting graft chains is a promising strategy to improve the performance of self-oscillating gels, it is still difficult to control the number of grafted chains. This is because of steric hindrance causing low macromonomer reactivity while introducing the graft chains.

Here, we succeeded in controlling the properties of grafted chains in self-oscillating gels by utilizing the "grafting from" method and ATRP. The base gel network was poly(NIPAAm- $r$-NAPMAm- $r$-NAPMAmRu(bpy) ${ }_{3}$ ), and the grafted chains were poly(NIPAAm) or poly (NIPAAm- $r$-NAPMAm- $r$-NAPMAmRu(bpy $\left.)_{3}\right)$. The controllability of ATRP for synthesizing poly(NIPAAm) and poly(NIPAAm- $r$-NAPMAm- $r$-NAPMAmRu(bpy $\left.)_{3}\right)$, which was achieved by varying the NAPMAm composition and ATRP reaction times (Table S1), was verified by the polymerization of the free polymers. As shown in Fig. 3 , the monomer conversion decreased when increasing 
the NAPMAm composition. The PDI values increased with the NAPMAm composition (Table S1). This phenomenon could be explained as follows: During ATRP, the metal-ligand catalyst promotes polymerization. However, monomers with primary amines could form a monomer-ligand complex, resulting in changed polymerization kinetics ${ }^{23}$. We utilized this property as a tradeoff between the NAPMAm composition and PDI values of the grafted chains. We attributed the NAPMAm compositions in the free polymers to the feeding ratio, despite the monomer conversion varying by changing the polymerization time, as shown in Table S1. These results implied that the composition and length of the grafted polymer chains in the comb-type gels were attributed to changing the feeding ratio and reaction time. We also verified that the graft polymer chains were successfully introduced in the whole gel region via ATRP using a fluorescent monomer probe (RhB) (Fig. S6). Finally, Ru (bpy) ${ }_{3}$-NHS was conjugated to the comb-type gels to fabricate comb-type self-oscillating gels.

To evaluate the self-oscillating performance of the comb-type self-oscillating gels, first, the equilibrium swelling behavior of the gels in their reduced and oxidized states was investigated. We proved that the increased composition of NAPMAm in the grafted chain resulted in a large difference in the equilibrium swelling ratio between the reduced and oxidized states (Fig. 4). We previously reported that the amount of $\mathrm{Ru}(\mathrm{bpy})_{3}$ in the self-oscillating gel (without grafted chains) affects the equilibrium swelling ratio ${ }^{13}$. Similarly, the amount of conjugated $\mathrm{Ru}(\mathrm{bpy})_{3}$ in the grafted chain influenced the equilibrium swelling ratio of the whole gel. As mentioned before, $\mathrm{Ru}(\mathrm{bpy})_{3}$-NHS reacts with the primary amine of NAPMAm ${ }^{24}$, and the NAPMAm contents in the Base Gel were consistent. Therefore, the amount of $\mathrm{Ru}(\mathrm{bpy})_{3}$ in the comb-type self-oscillating gels could depend on the composition of NAPMAm and the chain length of the graft chains. These results indicated that the graft chains affected the equilibrium swelling behavior. Thus, the swelling/deswelling kinetics of the gels in response to redox changes would be improved, owing to the more considerable differences in the equilibrium swelling ratios between the two redox states.

We demonstrated that the grafted chains with conjugated $\mathrm{Ru}(\mathrm{bpy})_{3}$ caused notable oscillation behavior compared with the Base Gel during the BZ reaction (Fig. 5). Although the grafted chain could accelerate the fast responsiveness of the whole gel network ${ }^{22}$, we concluded that the grafted chains without conjugated $\mathrm{Ru}(\mathrm{bpy})_{3}$ (the NINA_0 series) could not provide significantly changed oscillation behavior compared to the Base Gel. In contrast, the NINA_2 and NINA_10 series, which had conjugated $\mathrm{Ru}(\mathrm{bpy})_{3}$ in the grafted chains, showed improved self-oscillating behavior during the $\mathrm{BZ}$ reaction. When the
$\mathrm{Ru}(\text { bpy })_{3}$ in the gel is oxidized $\left(\mathrm{Ru}(\mathrm{bpy})_{3}{ }^{2+} \rightarrow \mathrm{Ru}\right.$ $(\text { bpy })_{3}{ }^{3+}$ ), the grafted chains with conjugated $\mathrm{Ru}(\mathrm{bpy})_{3}$ stretches rapidly due to the excluded volume effect and electrostatic repulsion. The fast responsiveness of the grafted chains with $\mathrm{Ru}(\mathrm{bpy})_{3}$ leads the whole gel to expand faster during the $\mathrm{BZ}$ reaction. Therefore, the comb-type self-oscillating gels of the NINA_2 and NINA_10 series swelled faster than those of the Base Gel and NINA_0 series. Likewise, when the $\mathrm{Ru}(\mathrm{bpy})_{3}$ in the gel is reduced $\left(\mathrm{Ru}(\mathrm{bpy})_{3}{ }^{3+} \rightarrow \mathrm{Ru}(\mathrm{bpy})_{3}{ }^{2+}\right)$, the grafted chains with conjugated $\mathrm{Ru}(\mathrm{bpy})_{3}$ exhibit fast contraction because of the hydrophobic interaction between the $\mathrm{Ru}$ (bpy) ${ }_{3}$ components in the grafted chain as well as the Base Gel network. The rapid contraction of the grafted chains induces the entire gel to shrink faster until the redox state is shifted ${ }^{14}$. The results of the NINA_2 and NINA_10 series clearly reflected the described contribution of conjugated $\mathrm{Ru}(\mathrm{bpy})_{3}$ in the grafted chain. In the NINA_2 series, the overall oscillation behavior was slightly enhanced but did not change with the ATRP reaction time. We believe that the lengths of the grafted chains were nearly the same regardless of the ATRP reaction time, which could be deduced from the freepolymer characterization (Fig. 3). In the NINA_10 series, the normalized amplitude increased with increasing ATRP reaction time, reflecting the increasing polymer length with increasing ATRP reaction time. Thus, controlling the ATRP reaction times led to the controlled oscillation behavior of the comb-type self-oscillating gels during the $\mathrm{BZ}$ reaction.

In this study, comb-type self-oscillating gels were successfully fabricated by immobilizing the ATRP initiator to the base poly(NIPAAm-r-NAPMAm) gel, followed by ATRP. As the composition of NAPMAm in the grafted chain and ATRP reaction time was increased, the difference in the equilibrium swelling ratio between the redox states increased. Moreover, the gels could swell/deswell faster with an enlarged amplitude during the BZ reaction. Taken together, these findings provide a strategy for achieving controlled oscillation behavior, which can be applied to biomimetic actuators.

\section{Acknowledgements}

This work was supported in part by the Grants-in-Aid for Scientific Research (No. 20H00388 to R.Y.) from the Ministry of Education, Culture, Sports, Science, and Technology of Japan. The authors are grateful to Mr. Taihei Nishimoto for kindly letting us use rhodamine B hydroxyethyl methacrylate ester (RhB), instructing the sample preparation method of RhB-grafted gels, and helping us use the fluorescence microscope for Fig. S3.

\section{Author contributions}

W.S.L. performed all experiments and authored the paper. T.E., A.M.A., and R.Y. conceived and directed the study as along with helping author the manuscript.

Conflict of interest

The authors declare no competing interests. 


\section{Publisher's note}

Springer Nature remains neutral with regard to jurisdictional claims in published maps and institutional affiliations.

Supplementary information The online version contains supplementary material available at https://doi.org/10.1038/s41427-022-00360-0.

Received: 6 October 2021 Revised: 24 December 2021 Accepted: 13 January 2022.

Published online: 18 February 2022

\section{References}

1. Liu, F. \& Urban, M. W. Recent advances and challenges in designing stimuliresponsive polymers. Prog. Polym. Sci. 35, 3-23 (2010).

2. Biomedical Applications of Hydrogels Handbook. 148, (Springer New York, 2010).

3. Yoshida, R., Takahashi, T., Yamaguchi, T. \& Ichijo, H. Self-oscillating gel. J. Am. Chem. Soc. 118, 5134-5135 (1996).

4. Yoshida, R. Self-oscillating polymer gels as biomimetic and smart softmaterials. Adv. Mater. Lett. 9, 836-842 (2018).

5. Maeda, S., Hara, Y., Sakai, T., Yoshida, R. \& Hashimoto, S. Self-walking gel. Adv. Mater. 19, 3480-3484 (2007).

6. Murase, Y., Maeda, S., Hashimoto, S. \& Yoshida, R. Design of a mass transport surface utilizing peristaltic motion of a self-oscillating gel. Langmuir $\mathbf{2 5}$ 483-489 (2009).

7. Tabata, O., Hirasawa, H., Aoki, S., Yoshida, R. \& Kokufuta, E. Ciliary motion actuator using self-oscillating gel. Sens. Actuators A Phys. 95, 234-238 (2002).

8. Shiraki, Y. \& Yoshida, R. Autonomous intestine-like motion of tubular selfoscillating gel. Angew. Chem. Int. Ed. 51, 6112-6116 (2012).

9. Yoshida, R., Takei, K. \& Yamaguchi, T. Self-beating motion of gels and modulation of oscillation rhythm synchronized with organic acid. Macromolecules 36, 1759-1761 (2003).

10. Ito, Y., Nogawa, M. \& Yoshida, R. Temperature control of the BelousovZhabotinsky reaction using a thermoresponsive polymer. Langmuir 19, 9577-9579 (2003).
11. Suzuki, D., Kobayashi, T., Yoshida, R. \& Hirai, T. Soft actuators of organized selfoscillating microgels. Soft Matter 8, 11447-11449 (2012).

12. Li, J., Li, X., Zheng, Z. \& Ding, X. A dynamic self-regulation actuator combined double network gel with gradient structure driven by chemical oscillating reaction. RSC Adv. 9, 13168-13172 (2019).

13. Masuda, T. et al. Control of swelling-deswelling behavior of a self-oscillating gel by designing the chemical structure. RSC Adv. 5, 5781-5787 (2015).

14. Mitsunaga, R., Okeyoshi, K., Yoshida, R. \& Li, T. Design of a comb-type selfoscillating gel. Chem. Commun. 49, 4935-4937 (2013).

15. Neugebauer, D., Zhang, Y., Pakula, T., Sheiko, S. S. \& Matyjaszewski, K. Denselygrafted and double-grafted PEO brushes via ATRP. A route to soft elastomers. Macromolecules 36, 6746-6755 (2003).

16. Coessens, V. M. C. \& Matyjaszewski, K. Fundamentals of atom transfer radical polymerization. J. Chem. Educ. 87, 916-919 (2010).

17. Matyjaszewski, K. Atom Transfer Radical Polymerization (ATRP): current status and future perspectives. Macromolecules 45, 4015-4039 (2012).

18. Paquin, F., Rivnay, J., Salleo, A., Stingelin, N. \& Silva, C. Multi-phase semicrystalline microstructures drive exciton dissociation in neat plastic semiconductors. J. Mater. Chem. C 3, 10715-10722 (2015).

19. Conradi, M. \& Junkers, T. Fast and efficient [2 + 2] UV cycloaddition for polymer modification via flow synthesis. Macromolecules 47, 5578-5585 (2014).

20. Chen, X., Wu, Q., Henschke, L., Weber, G. \& Weil, T. An efficient and versatile approach for the preparation of a rhodamine B ester bioprobe library. Dye Pigment. 94, 296-303 (2012).

21. Kaneko, Y. et al. Deswelling mechanism for comb-type grafted poly(N-isopropylacrylamide) hydrogels with rapid temperature responses. Polym. Gels Netw. 6, 333-345 (1998).

22. Yoshida, R. et al. Comb-type grafted hydrogels with rapid de-swelling response to temperature changes. Nature 374, 240-242 (1995).

23. Mendonça, P. V. et al. Straightforward ARGET ATRP for the synthesis of primary amine polymethacrylate with improved chain-end functionality under mild reaction conditions. Macromolecules 47, 4615-4621 (2014).

24. Ueki, T., Onoda, M., Tamate, R., Shibayama, M. \& Yoshida, R. Self-oscillating AB diblock copolymer developed by post modification strategy. Chaos $\mathbf{2 5}$ 064605 (2015). 